\title{
Patients $>40$ years with a glenohumeral dislocation have a lower incidence of Symptomatic Rotator Cuff Tear than previously reported and can be managed safely without routine advanced imaging
}

\section{Chibuzo Manafa}

Brighton and Sussex University Hospitals NHS Trust

Ahmed Barakat ( $\sim$ ahmedharoonbarakat@gmail.com )

Brighton and Sussex University Hospitals NHS Trust https://orcid.org/0000-0002-7742-7059

\section{Felicity Auld}

Brighton and Sussex University Hospitals NHS Trust Joideep Phadnis

Brighton and Sussex University Hospitals NHS Trust

\section{Research article}

Keywords: Primary shoulder dislocation, Rotator cuff tears, Virtual fracture clinic, Guidelines, Cost reduction, Oxford shoulder score

Posted Date: July 14th, 2020

DOI: https://doi.org/10.21203/rs.3.rs-39640/v1

License: (c) (i) This work is licensed under a Creative Commons Attribution 4.0 International License. Read Full License 


\section{Abstract}

Background Incidence of rotator cuff tears (RCT) following primary anterior glenohumeral dislocation in patients over 40 years is thought to be high. Current guidelines recommend specialist clinic review and advanced imaging for all patients. The aim of this study was to report the incidence of symptomatic RCT in a large cohort of patients and to assess the safety and efficacy of a physiotherapy led virtual protocol where patients were selectively investigated and treated based on symptoms.

Methods 238 consecutive patients over 40 years of age with a primary anterior glenohumeral dislocation were identified between January 2015 and June 2018 (42 months). All patients were managed initially through a physiotherapy led virtual clinic with selective referral of patients with red flag symptoms to a specialist shoulder clinic. Advanced imaging (MRI or USS) was performed at the discretion of the shoulder specialist following patient assessment. The incidence of symptomatic RCT was assessed and compared between two groups (Group 1: 40-70 years, Group 2: >70 years). All surgical interventions were recorded. Patients were assessed at a mean of 22 months (range 10-54) using the Oxford shoulder score; patient satisfaction and return to pre-injury functional level. Cost analysis was performed to compare management using this protocol versus published guidelines.

Results A total of 238 patients were identified with mean age of 65 years (range 40-96). 69\% ( $n=164 / 238$ ) were symptomatic and were referred to specialist clinic. The other $31 \%(n=74 / 238)$ were asymptomatic and were discharged to physiotherapy. Of those referred to a specialist clinic, only $46 \%$ ( $n=75 / 164)$ were sent for advanced imaging following clinical examination. The incidence of symptomatic RCTs was $31 \%$ $(n=51 / 164)$ in this group and only $22 \%(n=11 / 51)$ of those underwent subsequent surgical repair of their rotator cuff. Of those patients discharged to physiotherapy, $62 \%(n=47 / 74)$ responded to the questionnaire with $89 \%$ fully satisfied, $85 \%$ returned to pre-injury level and mean Oxford score was 42 (3338, SD 3.2) indicating excellent shoulder function. With all patients pooled excluding those lost to followup $(n=27)$, the incidence of symptomatic RCTs was $24 \%(51 / 211)$. The incidence in group 1 was $16 \%$ $(n=22 / 137)$ and was significantly lower than in group 2 which was $39 \%(n=29 / 74)(p<0.05)$. Cost analysis demonstrated $49 \%$ cost reduction using this protocol.

Conclusion Incidence of symptomatic cuff tears following a primary glenohumeral dislocation in patients over 40 years was lower than previously reported. A protocol based on selective referral and investigation for symptomatic patients was cost effective and safe.

\section{Introduction}

Rotator cuff tear (RCT) is a recognized complication of anterior shoulder dislocation, particularly with increasing age [1]. The incidence in patients between 40 and 60 years old is thought to be in the order of $40 \%$ with increasing frequency in patients $>60$ years old $[2,3]$. Guidelines such as those published by the British Elbow and Shoulder Society (BESS), recommend ultrasound scanning (USS) or Magnetic resonance imaging (MRI) and specialist shoulder clinic follow up for all patients over 40 years with an 
anterior dislocation [4]. Investigating and reviewing all patients in this scenario places significant financial and logistical burdens on the healthcare system especially as average life expectancy and activity level rises.

The presence of asymptomatic RCT in patients without a dislocation also rises with age, and hence whether tears found after dislocation are new, pre-existing or are the propagation of a pre-existing tear is uncertain. In a prospective comparative study by Berbig et al. [3] including 167 patients, it was found that the incidence of RCT after traumatic shoulder dislocation was $56 \%$ in patients 60 years or older compared to $44 \%$ in the control group of healthy matched volunteers. Simank et al. [2] studied 65 patients with a history of anterior glenohumeral dislocation over the age of 40 years reporting an incidence of $100 \%$ in patients older than 70 years and $56 \%$ for patients between 40 and 70 years. Interestingly, the authors found no difference in functional scores or outcome between patients with and without an RCT post dislocation, substantiating the notion that many of these tears are not symptomatic [2].

Hawkins et al. [4] reported on 61 patients older than 40 years after an anterior dislocation. Only $23 \%(\mathrm{n}=$ 14) had persistent pain and weakness after a trial of physiotherapy and were subsequently diagnosed with a RCT.

Hence, perhaps the emphasis should be on identifying symptomatic RCTs rather than RCTs per se, as most clinicians are unlikely to treat an asymptomatic cuff tear regardless of imaging features.

In our institution, all acute orthopaedic injuries not requiring in patient admission are initially managed remotely through a 'virtual clinic' model led by physiotherapists with consultant input. This has been demonstrated to improve efficiency and lower healthcare costs.

In the case of shoulder dislocation in patients over 40 years, only patients with ongoing symptoms beyond 3 weeks are referred for an appointment with a shoulder specialist. Imaging is then performed at the discretion of the clinician. The advantage of this model is lower cost, and reduction of the number of patients who need to attend a face to face specialist clinic in which appointments are at a premium.

It has been our anecdotal experience through this model that the number of patients with symptomatic RCTs requiring intervention is fewer than suggested in the literature and that only a small proportion of patients require advanced imaging following clinical review.

The primary aim of this study was to analyse the incidence of symptomatic RCTs in a large cohort of patients over 40 years of age with a traumatic primary anterior glenohumeral dislocation. The secondary aim was to assess the safety of managing this cohort using a virtual clinic model, with rationalization of clinic appointments and investigations.

\section{Methods}

The virtual fracture clinic (VFC) database was used to identify 431 consecutive patients with an isolated primary traumatic anterior shoulder dislocation between January 2015 and June 2018 (42 months). All 
Patients below 40 years of age $(n=193)$ were excluded, leaving a study population of 238 patients. Patients with a fracture dislocation or posterior dislocation were not included.

Patients were categorized into two age groups for comparison. Group 1 comprised patients between 40 and 70 years of age $(n=149)$ and Group 2 comprised patients over 70 years $(n=89)$. The age of 70 was chosen as a demarcation between the groups based on the previous evidence indicating a higher rate of RCTs in that age group [2].

A symptomatic RCT was defined as a patient with ongoing red flag symptoms of weakness, pain, stiffness or dysfunction on history or physical examination with evidence of a RCT on advanced imaging [5].

\section{Patient pathway}

Reduction of the initial glenohumeral dislocation is performed in the emergency department following antero-posterior and modified axillary radiographs. After radiographic confirmation that the shoulder is reduced, without evidence of a neurovascular injury, the patients are discharged with a broad arm sling applied. An electronic referral is made to the VFC.

The patient is telephoned the within 72 hours of referral by a specialist physiotherapist running the VFC. The patient is provided with a paper and electronic care plan that explains their injury, management, and exercises. Patients were also provided with an online link to a video demonstrating these exercises.

Patients were contacted at three weeks by VFC to screen for self-reported red flag signs such as painful motion, subjective weakness, or stiffness. The time point of three weeks was chosen for as it is known that persistence of weakness beyond this point is suggestive of a symptomatic RCT [5]. Patients with red flag symptoms were given a face to face appointment in a consultant led shoulder clinic within 6 weeks of their original injury. In the specialist clinic, the patient is examined for signs of a symptomatic rotator cuff tear. If the patient is progressing well, they are referred for physiotherapy without further routine review by a doctor. Patients where a symptomatic cuff tear is suspected are concurrently referred to physiotherapy and for advanced imaging (MRI or USS) at the discretion of the clinician (Fig. 1). Hence only a small proportion of patients see a shoulder specialist and a sub-group of these patients undergo further investigation/intervention.

The virtual clinic data base was retrospectively interrogated to analyse which patients had been referred to a specialist clinic and which had been managed with physiotherapy alone. The clinical records and imaging studies of the patients reviewed in a specialist clinic were analyzed to ascertain how they had been managed and how many had a symptomatic RCT diagnosed and treated.

The patients managed by physiotherapy without specialist review were contacted by phone and a questionnaire was sent via e mail or post to assess clinical outcome. The primary outcome measure used was the Oxford Shoulder Score (OSS). Secondary measures were return to pre-injury level of function and 
sports if applicable; patient satisfaction (fully, partly satisfied, or unsatisfied) and whether they had attended another institution for management of their shoulder subsequent to their dislocation.

A cost analysis using recently published UK government healthcare costing data was performed to compare the actual cost of treatment through the outlined management protocol versus the cost of investigating every patient with an MRI scan and follow up in a consultant led clinic [6].

\section{Ethics statement}

This study protocol has been approved by the Institutional Review Board (IRB) of Brighton and Sussex University Hospitals - UK and that it conformed to the tenets of the Declaration of Helsinki with informed consent obtained from all patients for research publication purposes.

\section{Results}

There were 124 male patients and 114 female patients. The mean age was 65 years (range $40-96$ years). There were 149 patients in group 1 (40-70 years) and 89 patients in group 2 ( $>70$ years).

All patients were initially reviewed through the VFC and referred to physiotherapy. Only those patients with red flag signs indicating possible symptomatic RCT after three weeks (as reported by physiotherapy follow-up or by patients themselves contacting the VFC) were referred to a specialist clinic. 74/238 patients (31\%) were discharged from the VFC to be managed by physiotherapy as they remained asymptomatic after three weeks and did not need further imaging or specialist consultation. 48 of these (65\%) were in group 1 and 26 were in group 2 (35\%).

$164 / 238(69 \%)$ patients required review in a specialist shoulder clinic because of ongoing symptoms or dysfunction at three weeks. Of those, $89 / 164$ patients (54\%) were discharged to physiotherapy following clinical assessment by a specialist shoulder surgeon without further investigation of their shoulder as no objective signs or weakness indicating a symptomatic RCT was elicited on clinical examination. None of the patients referred to physiotherapy following specialist clinic assessment were referred back to the specialist clinic because of failure to progress. The remaining 75/164 patients (46\%) underwent further radiological investigation at the clinician's discretion in the form of Magnetic resonance imaging (MRI) (n $=35)$ or Ultrasound scan (USS) $(n=40)$.

The incidence of a symptomatic RCT in the group of patients seen in a specialist shoulder clinic was $31 \%$ $(51 / 164$ patients). The incidence was significantly lower $(p=<0.001)$ in Group $1((22 / 102) 22 \%)$ compared to Group 2 ((29/62) 47\%).

Of the 51 patients identified with a symptomatic RCT as per their clinical examination and advanced imaging, $11(22 \%)$ underwent subsequent surgical repair of their rotator cuff. eight of these patients had an open rotator cuff repair; two had reverse shoulder replacement while one of the patients had an arthroscopic rotator cuff repair. The other patients were successfully treated with injections and physiotherapy after shared decision making with the consultant. 
Of the patients discharged by physiotherapy and were not evaluated by a specialist, 47/74 (62\%) of them were contactable and responded to the questionnaire. Three were deceased; seven had dementia; six were overseas with no contact details and 11 declined to complete the questionnaire. None of the patients contacted required further consultations or subsequent surgery. $42 / 47$ (89\%) patients were fully satisfied with treatment and $40 / 47$ (85\%) who participated in sports prior to injury had returned to sports at their previous level. The mean and median Oxford Shoulder Score was 42 and 44 respectively (range 33-48) indicating good or excellent shoulder function in most patients.

With all patients pooled excluding those not contactable or assessable $(n=211)$, the incidence of symptomatic RCT was $24 \%$ (51/211). The incidence in group 1 ((22/137), 16\%) was significantly lower than in group $2((29 / 74) 39 \%)(p<0.05)$ (Fig. 2$)$.

\section{Cost Analysis}

This was performed based on the cost of a new patient attending a general fracture clinic (£168) versus a virtual (£64) clinic while an advanced imaging would cost on average $£ 152(£ 272$ for MRI arthrogram and $£ 47$ for a shoulder USS) [6, 7]. A cost analysis revealed that if in accordance to the recent guidelines, all patients above 40 years in this cohort were referred to a specialist clinic with further advanced imaging, the total costs would be $£ 76,160$. Our management protocol costed $£ 38,952$ (49\% cost reduction) where VFC model was used for initial assessment and only patients with suspected symptomatic RCT were referred to a specialist clinic for further investigations and evaluation.

\section{Discussion}

In this study, it was demonstrated that the incidence of symptomatic RCTs requiring surgery or resulting in disability was lower than reported previously in the literature. In addition, patients were able to be managed safely with selective investigation and specialist review based on symptoms rather than protocol. The overall reported incidence of clinically and radiologically proven symptomatic RCT was lower (31\%) than in some other studies many of which are based on smaller numbers of patients. Pevny et al. [8] reported a series of 52 dislocations in patients older than age 40 and found that $18(35 \%)$ had evidence of RCT although it was unclear if these were all symptomatic or not, while Neviaser et al. [9] and Ribbans et al. [10] reported an incidence of $86 \%$ and $61 \%$ symptomatic tears respectively .

The discrepancy in incidence may be due to the reporting of asymptomatic RCT in some studies. The incidence of asymptomatic RCTs is known to increase with age in the general population and diagnosis of these tears following a dislocation may be misinterpreted as an acute event. In a prospective controlled study of 167 patients Berbig et al. [3] found that the incidence of RCT following shoulder dislocation was $56 \%$ in patients $>60$ years however it was also noted that the incidence of asymptomatic RCT in their control group was $44 \%$. It was hence of greater interest to us to understand the rate of symptomatic RCT as this is more influential on patient management. 
Using the pathway described through triage of symptomatic patients by physiotherapy only $69 \%$ of patients underwent early evaluation by a shoulder specialist. Of these, only $31 \%$ were diagnosed with a symptomatic RCT on imaging and a large proportion (54\%) were referred for physiotherapy without further investigation. This practice of individualized patient assessment is advocated by other studies looking at the outcome of patients with RCT after dislocation. Hawkins et al. [4] reported on 61 patients > 40 years after an anterior dislocation. $23 \%(n=14)$ had persistent pain and weakness after a trial of physiotherapy and were subsequently diagnosed with a RCT, meaning that $77 \%$ did not require intervention following a period of physiotherapy. Similarly, Sonnabend [11] identified a subgroup of 13/27 patients with persistent pain and weakness 3 weeks after dislocation. The asymptomatic patients did not undergo further investigation and of the 27 patients who were symptom free at 3 weeks, only four had ongoing pain or dysfunction at 3 months indicating the majority of patients will improve in the first three months.

It was also of interest to stratify the risk of RCT by age in our cohort of patients. There was a significantly higher incidence of symptomatic RCT in patients $>70$ years as compared to those between 40 and 70 years. This correlates with the increasing incidence of asymptomatic RCTs in the general population which has been shown to be up to $100 \%$ in individuals in their eighties and nineties [12] and $56 \%$ for patients between 40 and 70 years. The proposed mechanism of why patients $>40$ years are at risk of RCT is that with advancing age the posterior and superior cuff becomes less pliable and more liable to be torn during a dislocation [13]. However, to our best knowledge it has not been investigated whether a preexisting RCT is actually a risk factor for dislocation. Many patients sustain an acute RCT following a fall without a dislocation hence it could be considered that an intact rotator cuff is protective against dislocation, whereas a pre-existing tear of the rotator cuff may allow a dislocation to occur. Those patients that have symptomatic RCTs after a dislocation are more likely to have acute on chronic tears that have propagated rather than occurred afresh [14-16].

\section{Limitations}

A limitation of this study was that 27 patients (11\%) were lost to follow up or declined to participate. Consequently, we cannot be sure that the incidence of symptomatic RCT was not higher, however we do know that these patients were all discharged to physiotherapy because of good early outcome without red flag signs. We are reassured by Sonnabends data that it is uncommon that these patients will deteriorate beyond three months[11]. Moreover, none of the other patients discharged early to physiotherapy $(n=47)$ reported a deterioration in their symptoms or treatment/investigation at another institute when contacted. Another limitation may be that not every patient following dislocation underwent USS or MRI, therefore some RCTs may not have been diagnosed, however as stated previously we feel that symptomatic tears are of more clinically relevant.

On the other hand, the protocol followed was based on the identification of ongoing symptoms and altered function, to determine investigation and treatment. This makes the assumption that the specialist shoulder surgeons did not underdiagnose or miss the presence of symptoms. 
The protocol described is in place because of the well-established virtual clinic in our institute which has pioneered the VFC concept in the UK [17]. The cost savings achieved and described are hence a reflection of what would be saved if the national guidelines for management of these patients was followed rigidly. A limitation is that other units may not be able to replicate the treatment protocol without the VFC system in place, however we feel that the essence of individualized patient decision making for this pathology is still applicable.

\section{Conclusion}

Based on the results of this study we would advocate that patients $>40$ years are assessed on an individualized basis in order to determine the need for further investigation and treatment and that clinicians are cognizant that many RCTs found after a dislocation may be pre-existing and asymptomatic in the long term.

\section{Abbreviations}

RCT : rotator cuff tear

VFC : virtual fracture clinic

MRI : magnetic resonance imaging

USS : ultrasound scan

\section{Declarations}

\section{Ethics approval and consent to participate}

The authors declare that institutional review board (IRB) approval at Brighton and Sussex university hospitals - UK was granted for this research.

\section{Consent for publication}

An informed consent was granted from all participants for publication purposes.

\section{Availability of data and materials}

The authors will give access to all data if requested

\section{Competing interests}

All authors declare that they have no competing interests.

\section{Funding}


All authors declare that no funds were received for this research.

\section{Authors' contributions}

FA provided the patient's data. $\mathrm{AB}$ and $\mathrm{CM}$ analyzed the patient's data and were major contributors in writing the manuscript. JP contributed in the final drafting of the manuscript. All authors read and approved the final manuscript.

\section{Acknowledgements}

None declared.

\section{References}

1. McLaughlin MC. Trauma. Saunders, Philadelphia. 1960;pp233-296.

2. Simank HG, Dauer G, Schneider S, Loew M. Incidence of rotator cuff tears in shoulder dislocations and results of therapy in older patients. Arch Orthop Trauma Surg. 2006;126:235-240.

3. Berbig, R., et al., Primary anterior shoulder dislocation and rotator cuff tears. J Shoulder Elbow Surg, 1999; 8(3): 220-5.

4. Hawkins RJ, Bell RH, Hawkins RH, Koppert GJ. Anterior dislocation of the shoulder in the older patient. Clin Orthop Relat Res. 1986;206:192-195.

5. Gomberawalla, M. M., \& Sekiya, J. K. (2013). Rotator Cuff Tear and Glenohumeral Instability. Clinical Orthopaedics and Related Research ${ }^{\circledR}, 472(8), 2448-2456$.

6. Retrieved 26 October 2019, from https://improvement.nhs.uk/documents/4981/AnnexA_1920_National_tariff_workbook.xlsx

7. Freeman, R., Khanna, S., \& Ricketts, D. (2013). Inappropriate requests for magnetic resonance scans of the shoulder. International Orthopaedics, 37(11), 2181-2184.

8. 8. Pevny T, Hunter RE, Freeman JR. Primary Traumatic Anterior Shoulder Dislocation in Patients 40 Years of Age and Older. Arthroscopy. 1998;14(3): 289-294.

9. Neviaser RJ, Neviaser TJ, Neviaser JS. Anterior Dislocation of the Shoulder and Rotator Cuff Rupture. Clin Orthop Relat Res. 1993;291: 103-106.

10. Ribbans WJ, Mitchell R, Taylor GJ. Computerised Arthrotomography of Primary Anterior Dislocation of the Shoulder. J Bone Joint Surg Br. 1990;72(2): 181-185.

11. Sonnabend DH. Treatment of primary anterior shoulder dislocation in patients older than 40 years of age: conservative versus operative. Clin Orthop Relat Res. 1994;304:74-77

12. .Milgrom C, Schaffler M, Gilbert S, van Holsbeeck M. Rotator-cuff changes in asymptomatic adults. The effect of age, hand dominance and gender. J Bone Joint Surg Br. 1995;77(2):296- 298.

13. Toolanen G, Hildingsson C, Hedlund T, Knibestol M, Oberg L. Early Complications after Anterior Dislocation of the Shoulder in Patients over 40 Years. Acta Orthop Scand. 1993;64(5): 549-552. 
14. Sher JS, Uribe JW, Posada A, Murphy BJ, Zlatkin MB. Abnormal findings on magnetic resonance images of asymptomatic shoulders. J Bone Joint Surg Am. 1995;77:10-5.

15. Schibany N, Zehetgruber H, Kainberger F, Wurnig C, Ba-Ssalamah A, Herneth AM, Lang T, Gruber D, Breitenseher MJ. Rotator cuff tears in asymptomatic individuals: a clinical and ultrasonographic screening study. Eur J Radiol. 2004;51: 263-8.

16. Tempelhof S, Rupp S, Seil R. Age-related prevalence of rotator cuff tears in asymptomatic shoulders. J Shoulder Elbow Surg. 1999;8:296-9.

17. Holgate J, Kirmani S, Anand R. Virtual fracture clinic delivers British Orthopaedic Association compliance. Ann R Coll Surg Engl. 2016;22:1-4.

\section{Figures}

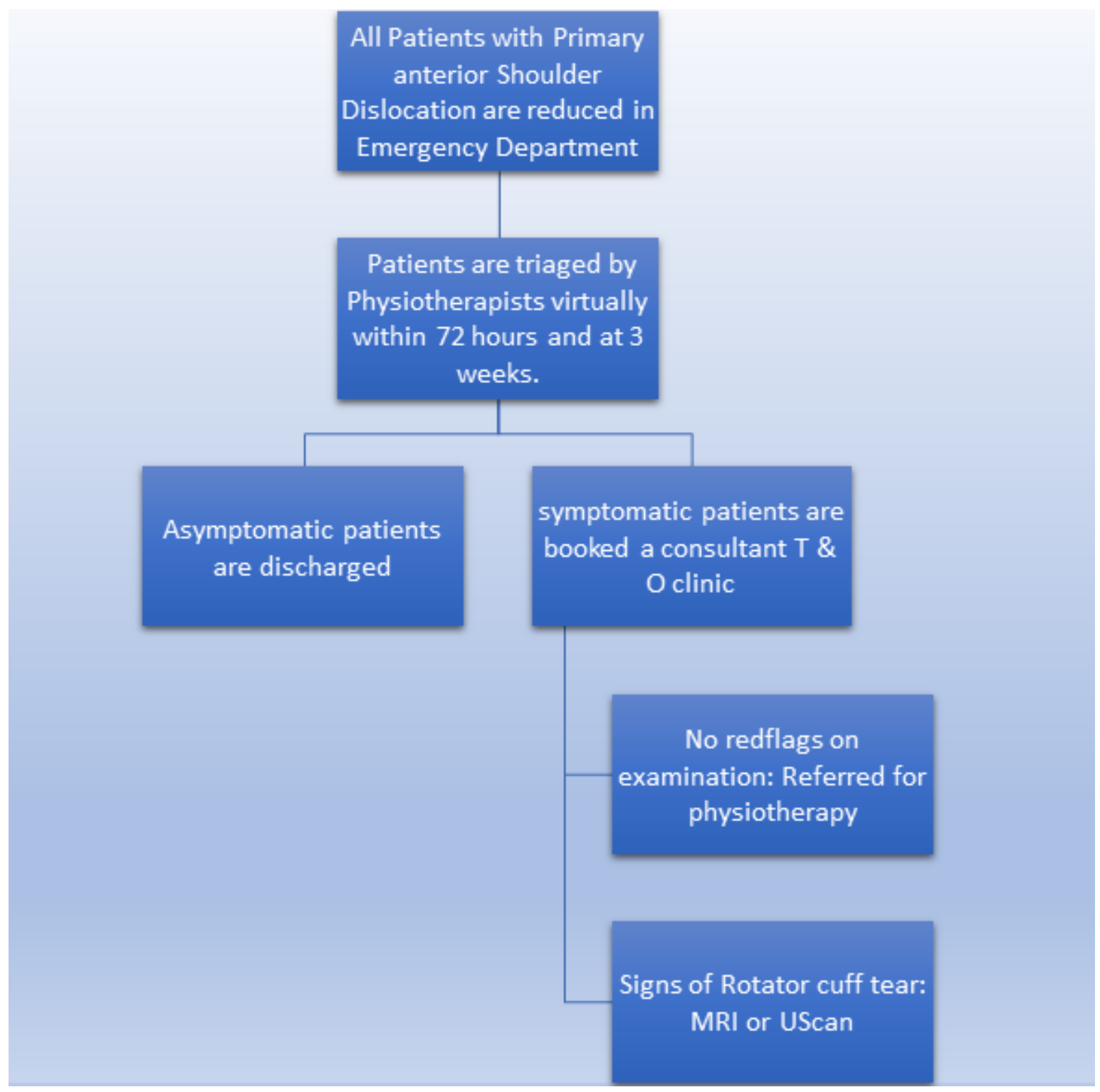

Figure 1 
A flow diagram outlines the flow of patients through the outlined management protocol.

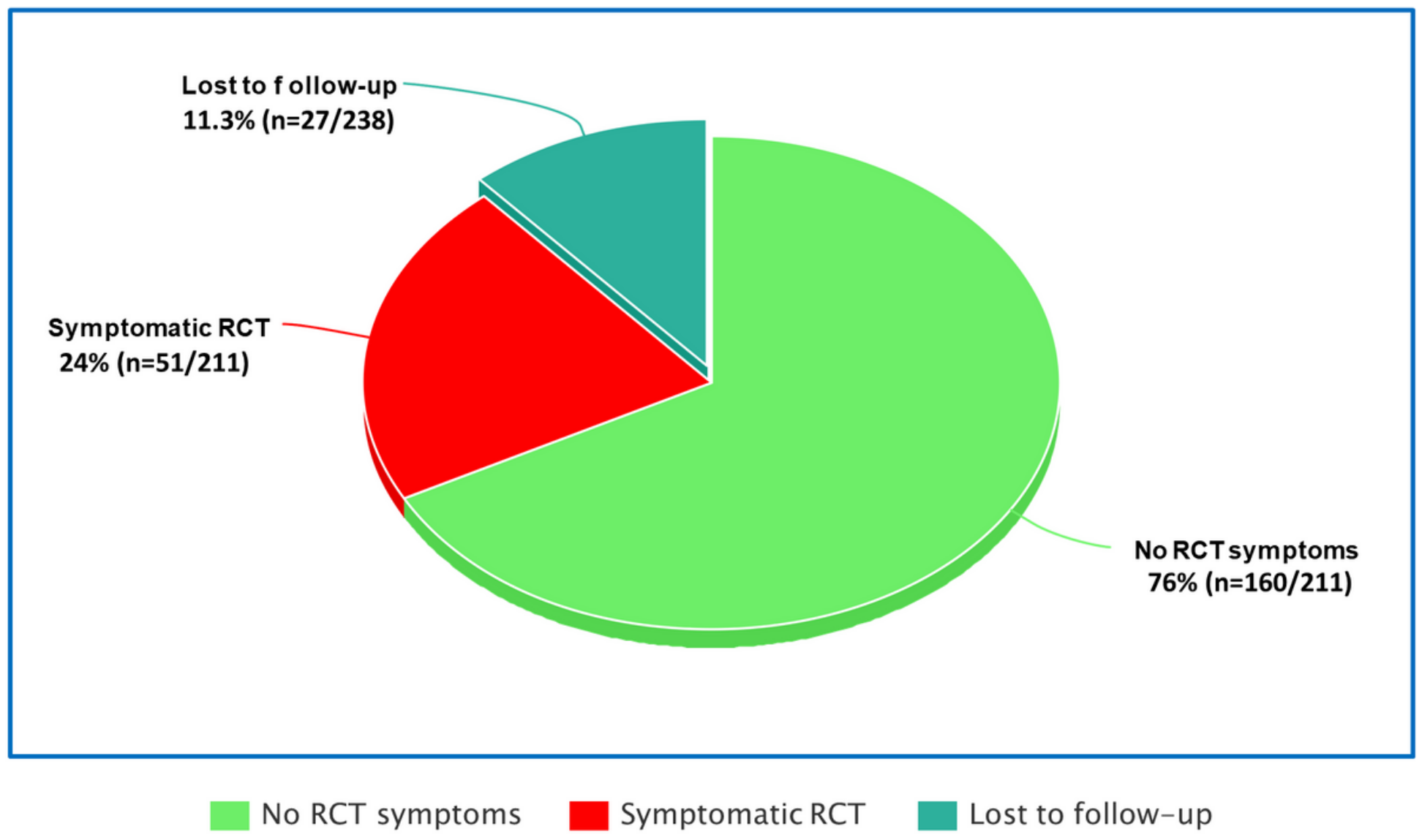

\section{Figure 2}

A pie chart depicting that out of all patients excluding those lost to follow-up, the overall incidence of symptomatic RCT proven clinically or radiologically was only $24 \%$. 